\title{
Verticilliose de la tomate : analyse des interac- tions entre l'agressivité de l'agent pathogène et la résistance générale de l'hôte
}

\author{
Issa BEYE \& Jean-François LAFAY \\ Laboratoire de Cryptogamie associé au C.N.R.S. (UA.086), Université Paris-Sud, Bâtiment 400, F 91405 \\ Orsay Cedex
}

RÉSUMÉ

\begin{abstract}
La résistance générale de la tomate à la verticilliose peut être analysée en termes de composantes indépendantes. Les composantes suivantes ont été étudiées chez 4 variétés de tomate ("Raf », "Supermarmande ", " Red Jacket » et " Marmande VR ») auxquelles on a inoculé 5 lignées apparentées de Verticillium dahliae : durée de la période d'incubation, extension des symptômes foliaires sur la tige, intensité des altérations foliaires, troubles de la croissance, de l'émission des feuilles et des fleurs.

Les 4 variétés de tomate se sont révélées sensibles, et pour tous ces paramètres on constate une interaction entre variété de l'hôte et lignée du parasite et l'on définit des composantes complémentaires, d'agressivité chez le parasite et de résistance chez l'hôte.

On discute des similitudes avec l'interaction résistance spécifique - virulence et des conséquences possibles de ce schéma au niveau des populations de parasite.
\end{abstract}

Mots clés additionnels : Composantes, notations, incubation, symptômes foliaires, croissance, floraison.

Verticillium wilt of tomato : analysis of interactions between pathogen aggressiveness and general host resistance.

Five related strains of Verticillium dahliae were inoculated into four susceptible varieties of tomato ("Raf", "Supermarmande", "Red Jacket" and "Marmande VR"), and the following quantitative aspects of the disease were recorded : duration of the incubation period, extent of leaf symptoms along the stem, intensity of leaf damage, disturbance in growth, leaf development and flowering. The value of these parameters depended on both the pathogen strain and the host variety, i.e. there was a host $x$ variety interaction. These parameters were, therefore, taken as distinct expressions, or discrete components, of the general resistance of the host. Reciprocally, they could also be viewed as discrete components of pathogen aggressiveness. This situation of complementary interacting components parallels the interaction between the specific resistance of a host and the virulence of a pathogen. This similarity and its possible implications on pathogen populations are discussed.

Additional key words : Components, scoring, incubation, foliar symptoms, growth, flowering.

\section{INTRODUCTION}

Le flétrissement vasculaire causé par Verticillium dahliae Kleb. est une maladie complexe qui comporte plusieurs séquences d'événements lors de l'installation du parasite et de l'exercice de son pouvoir pathogène. $\mathrm{Au}$ cours de ces séquences se produisent entre l'hôte et le parasite des interactions dont l'effet se traduit finalement par la gravité de la maladie.

Les interactions au niveau des pathosystèmes sont bien décrites dans le cadre de la relation entre la virulence et la résistance spécifique. C'est ainsi qu'après avoir été qualifiée de qualitative, essentiellement basée sur la relation gène pour gène de FLOR (1956), il est maintenant admis, à la lumière de travaux tels que ceux de CROSSE (1975) sur le couple Pseudomonas mors-prunorum Wormald - cerisier et ceux de CLIFFORD \& CLOTHIER (1974) sur le couple Puccinia hordei Otth. - orge, que la résistance spécifique peut s'exprimer de façon quantitative. En effet, ces 2 derniers auteurs ont montré l'existence d'une interaction hautement significative entre cultivars d'orge et souches de Puccinia si l'on prenait comme critère le nombre d'urédospores produit par $\mathrm{cm}^{2}$ de surface foliaire, ce qui 
n'empêche pas la réussite de l'inoculation dans tous les cas expérimentés. Pour ce qui a trait à l'interaction exprimée quantitativement et non plus qualitativement entre l'agressivité et la résistance générale, des travaux suggèrent qu'elle pourrait présenter les caractères d'une somme d'interactions spécifiques entre les gènes les contrôlant. Dans le cas du couple Phytophthora infestans (Mont.) de Bary - pomme de terre, CHEVAUGEON (1979) suggérait que la résistance générale de la pomme de terre au Mildiou pouvait résulter de la combinaison d'effets polygéniques spécifiques qui sont susceptibles d'exercer une pression sur la population parasite et orienter son évolution. MARTIN \& ELINGBOE (1976) lors de leur étude sur Erysiphe graminis DC. f. sp. tritici E. Marchal sur le blé ont démontré que la résistance horizontale pouvait résulter d'un ensemble d'interactions gène pour gène mais il s'agirait de gènes mineurs qui n'instaurent donc pas une compatibilité ou une incompatibilité complète entre hôte et pathogène. A la différence de ces 2 derniers auteurs, PARLEVLIET \& ZADOKS (1977) ont fait une étude théorique et sont allés dans le même sens en parlant de gènes participant à une résistance polygénique de l'hôte et s'exprimant, selon une relation gène pour gène, face à des gènes complémentaires déterminant l'agressivité du parasite. Le modèle proposé par PAR LEVLIET \& ZADOKS (1977) met bien en évidence la différence entre les modèles classiques, strictement additifs et un modèle strictement interactif. Il permet de mieux comprendre l'expression quantitative des relations spécifiques.

Enfin, SKAJENNIKOFF \& RAPILLY (1983) décrivent 2 sortes d'interaction dans le pathosystème Septoria nodorum Berk. - blé si l'on considère la période d'incubation. La première s'exerce entre différentes souches du champignon et différentes variétés de blé, la seconde entre souches et organes attaqués. Ils concluent que les systèmes polygéniques de l'hôte ne sont pas neutres ; ils font subir à la structure des populations pathogènes une pression de sélection donnant l'avantage aux individus les plus aptes à les surmonter. En tenant compte de ces faits expérimentaux, observations et études théoriques, on pourrait se demander ce qu'il en est dans le cas de la verticilliose de la tomate. La résistance générale de la tomate ne présente-t-elle pas, à chacune des étapes du cyle infectieux ou au niveau de chacun des multiples effets de $V$. dahliae sur la plante, des interactions spécifiques ? On est d'autant plus fondé à se poser cette question que, d'une part, SEBTI (1982) avait montré l'existence chez le pathogène de composantes d'agressivité qui peuvent varier irı́épendamment et que, d'autre part, selon BEYE \& LAFAY (1985), il n'y a pas, chez la plante, de relations constantes et univoques entre les différentes formes sous lesquelles s'exprime la verticilliose de la tomate. Ces derniers auteurs ont en effet établi qu'un haut niveau de résistance aux effets du parasite sur l'émission des feuilles par exemple ne va pas nécessairement de pair avec un haut niveau de résistance aux effets sur la photosynthèse, effets mesurés par l'intensité des altérations foliaires.

Pour le pathosystème que nous étudions, y a-t-il des interactions entre les composantes d'agressivité et celles de la résistance générale et, si oui, lesquelles? Ces interactions sont-elles valables pour tous les couples ou interviennent-elles uniquement pour certaines souches vis-à-vis de certaines variétés ? Les couples interagissent-ils au niveau des mêmes composantes ?

Pour répondre à ces questions, nous avons inoculé 5 souches de $V$. dahliae à 4 variétés de tomate puis mesuré l'évolution de la maladie.

Le choix des variétés s'est appuyé sur les différences observées antérieurement, dans leurs réponses à l'inoculation de l'une des souches (BEYE \& LAFAY, 1985). Quant aux souches de $V$. dahliae, elles étaient déjà connues pour leurs différences d'aptitude à provoquer chez la variété «Supermarmande » diverses manifestations de la verticilliose (SEBTI, 1982).

Les divers effets de l'agressivité et de la résistance générale peuvent être pris en compte en décrivant et en quantifiant les différentes manifestations de la maladie. La détection d'interactions spécifiques entre composantes de l'agressivité et de la résistance générale exige des analyses complémentaires. VAN DER PLANK (1978) et PARLEVLIET (1976) proposent d'effectuer des classements puis, si des anomalies de classement suggèrent l'existence d'interactions souche $\times$ variété, de procéder à une analyse de variance pour le vérifier. C'est la démarche que nous avons suivie dans le présent travail.

\section{MATÉRIEL ET MÉTHODES}

\section{A. L'agent pathogène}

L'isolat initial de Verticillium dahliae a été obtenu en 1978 à partir d'une tige de tomate atteinte de verticilliose dans la région de Casablanca (Maroc).

On a retenu le clone $\mathrm{C} 19$ dérivé de cet isolat initial, et qui présente les caractéristiques indiquées par BEYE \& LAFAY (1985), ainsi que 4 lignées recombinées («5/315», «211/315», «76/211», « 156/160») isolées et clonées par SEBTI (1982) à partir d'hétérocaryons associant des mutants du clone C19. Selon SEBTI (1982) ces lignées sont très probablement haplö̈des et issues de recombinaisons mitotiques. Aucune d'entre elles n'a montré exactement le même comportement que le clone sauvage " $\mathrm{C} 19$ », qu'elle soit inoculée à de jeunes plantes pourvues d'une seule feuille vraie, ou à des plantes plus âgées portant 5 feuilles déployées.

Toutes ces souches avaient été conservées dans l'azote liquide avant d'être reprises et utilisées.

\section{B. L'hôte, inoculation, notations, analyses statistiques}

Les semences des 4 variétés (« Raf », « Marmande $V R$ » qui possède le gène $V e$ de résistance spécifique à la race commune de $V$. dahliae, "Supermarmande » et " Red Jacket » ou "Casaque rouge ») ont été aimablement fournies par les Ets CLAUSE. Les conditions d'obtention des plantes, le mode d'inoculation (application d'une suspension de spores sur des radicelles blessées) et la notation des effets de l'inoculation sont ceux décrits par BEYE \& LAFAY (1985). Chacune des 5 lignées de Verticillium a été inoculée à 36 plantes de chacune des 4 variétés de tomate. Les différentes 
expressions de la maladie retenues sont : la durée de la période d'incubation, l'extension des symptômes sur la tige, l'intensité des altérations foliaires, la croissance de l'axe aérien, le nombre de feuilles émis et le nombre de fleurs des 3 premiers bouquets floraux.

Le logiciel statistique SPSS (Statistical Package for the Social Sciences) a été utilisé pour l'analyse des résultats (Univac 1190, Centre Paris-Sud Informatique).

Dans le cas des paramètres mesurés 6 fois dans le temps pour décrire leur évolution, les résultats figurent sur des graphiques portant des lettres qui représentent le classement par le test de rang de DunCAN (1955) des valeurs moyennes relatives à une date d'observation. Par contre, les résultats des paramètres mesurés une seule fois figurent sur les tableaux 1 et 2 comprenant le test de rang des moyennes et l'analyse de variance. De plus, dans toutes les variétés, certaines plantes n'ont pas montré clairement de symptômes foliaires de verticilliose pendant la durée de l'expérience. Elles ne sont pas prises en compte pour les statistiques relatives à la période d'incubation, mais sont quand même observées et notées pour tous les autres paramètres, et incluses dans les statistiques correspondantes. On a vérifié que cette façon d'opérer, bien que déplaçant les moyennes, ne modifiait pas significativement les classements auxquels on se réfère.

\section{RÉSULTATS}

\section{A. Durée de la période d'incubation (tabl. 1)}

Si l'on considère la durée de la période d'incubation comme l'une des expressions de l'agressivité de $V$. dahliae, une même lignée peut se comporter comme la plus agressive des 5 vis-à-vis d'une variété et la moins agres-

\section{TABLEAU 1}

Période d'incubation (en jours), pour les 390 plantes ayant présenté des symptômes. Duration (in days) of the incubation period, for the 390 plants which showed symptoms.

\section{TABLEAU la}

Test de rang de DunCAN (niveau 5 . 100) des durées moyennes, en fonction des variétés et des lignées. L'effectif de chaque classe est entre parenthèses.

$\mathrm{a}, \mathrm{b}, \mathrm{c}, \mathrm{d}$ : classement des lignées par variété : à lire verticalement.

A, B, C : classement des variétés par lignée : à lire horizontalement.

DUNCAN multiple range test (5\% level) of average duration for different varieties and strains. Class size in brackets. $a, b, c, d$ : classification of strains by variety: read vertically.

A, B, C : classification of varieties by strain : read horizontally.

\begin{tabular}{|c|c|c|c|c|c|c|c|c|}
\hline \multirow[t]{2}{*}{ Lignées } & \multicolumn{8}{|c|}{ Variétés } \\
\hline & & Raf & & ermarmande & & d Jacket & & mande VR \\
\hline $\mathrm{C} 19$ & $c$ & $\begin{array}{c}\text { B } \\
14,2 \pm 0,6 \\
(24)\end{array}$ & b & $\begin{array}{c}\mathrm{B} \\
14,8 \pm 0,7 \\
(29)\end{array}$ & b & $\begin{array}{l}\mathrm{B} \\
15,5 \pm 0,9 \\
(21)\end{array}$ & a & $\begin{array}{l}\mathrm{A} \\
19,0 \pm 1,1 \\
(27)\end{array}$ \\
\hline $76 / 211$ & d & $\begin{array}{l}C \\
7,9 \pm 0,7 \\
(17)\end{array}$ & a & $\begin{array}{c}\mathrm{A} \\
20,2 \\
(23)\end{array}$ & $a b$ & $\begin{array}{l}\text { B } \\
17,4 \pm 1,6 \\
(14)\end{array}$ & a & $\begin{array}{l}\mathrm{A} \\
19,6 \pm 1,5 \\
(24)\end{array}$ \\
\hline $5 / 315$ & b & $\begin{array}{c}\mathrm{B} \\
16,3 \pm 1,2 \\
(22)\end{array}$ & b & $\begin{array}{l}\mathrm{B} \\
16,0 \pm 1,0 \\
(26)\end{array}$ & a & $\begin{array}{l}\mathrm{A} \\
18,9 \pm 1,4 \\
(17)\end{array}$ & b & $\begin{array}{l}\mathrm{B} \\
15,7 \pm 2,7 \\
(10)\end{array}$ \\
\hline $211 / 315$ & a & $\begin{array}{l}\mathrm{A} \\
18.0 \pm 1.8 \\
(21)\end{array}$ & b & $\begin{array}{c}\mathrm{A} \\
16,3 \pm 1,6 \\
(20)\end{array}$ & $\mathrm{c}$ & $\begin{array}{l}\mathrm{B} \\
7,0 \pm 3,5 \\
(22)\end{array}$ & $a b$ & $\begin{array}{c}\text { B } \\
17,7 \pm 2,6 \\
(16)\end{array}$ \\
\hline $156 / 160$ & $\mathrm{c}$ & $\begin{array}{c}\mathrm{C} \\
14,0 \pm 0,4 \\
(22)\end{array}$ & $\mathrm{a}$ & $\begin{array}{c}\mathrm{A} \\
20,5 \pm 2,2 \\
(10)\end{array}$ & $a b$ & $\begin{array}{l}\text { B } \\
17,1 \pm 2,7 \\
(12)\end{array}$ & $a b$ & $\begin{array}{l}\mathrm{AB} \\
18,2 \pm 1,9 \\
(13)\end{array}$ \\
\hline
\end{tabular}

TABLEAU $1 \mathrm{~b}$

Analyse de variance à 2 critères de classification (variété $\times$ lignée).

$n:$ nombre de variétés de tomate; $p:$ nombre de lignées de Verticillium.

Two way analysis of variance ( $n$ varieties $\times$ ptrains).

\begin{tabular}{cccccc}
\hline Source de variation & Degrés de liberté & Carré moyen & F & Signification de F \\
\cline { 2 - 6 } & 3 & $(\mathrm{n}-1)$ & 373,215 & 30,58 & $<0,001$ \\
Variété & 4 & $(\mathrm{p}-1)$ & 73,689 & 6,038 & $<0,001$ \\
Lignée & 12 & $(\mathrm{n}-1)(\mathrm{p}-1)$ & 269,708 & $<0,001$ \\
Variété $\times$ lignée & 19 & $(\mathrm{np}-1)$ & 245,483 & 22,099 & $<0,001$ \\
Total expliqué & 370 & 12,205 & 20,114 & \\
Résiduelle & & 370 & & \\
\hline \hline
\end{tabular}


EXTENSION DES SYMPTOMES SUR LA TIGE

EN \% DE LA TAILLE

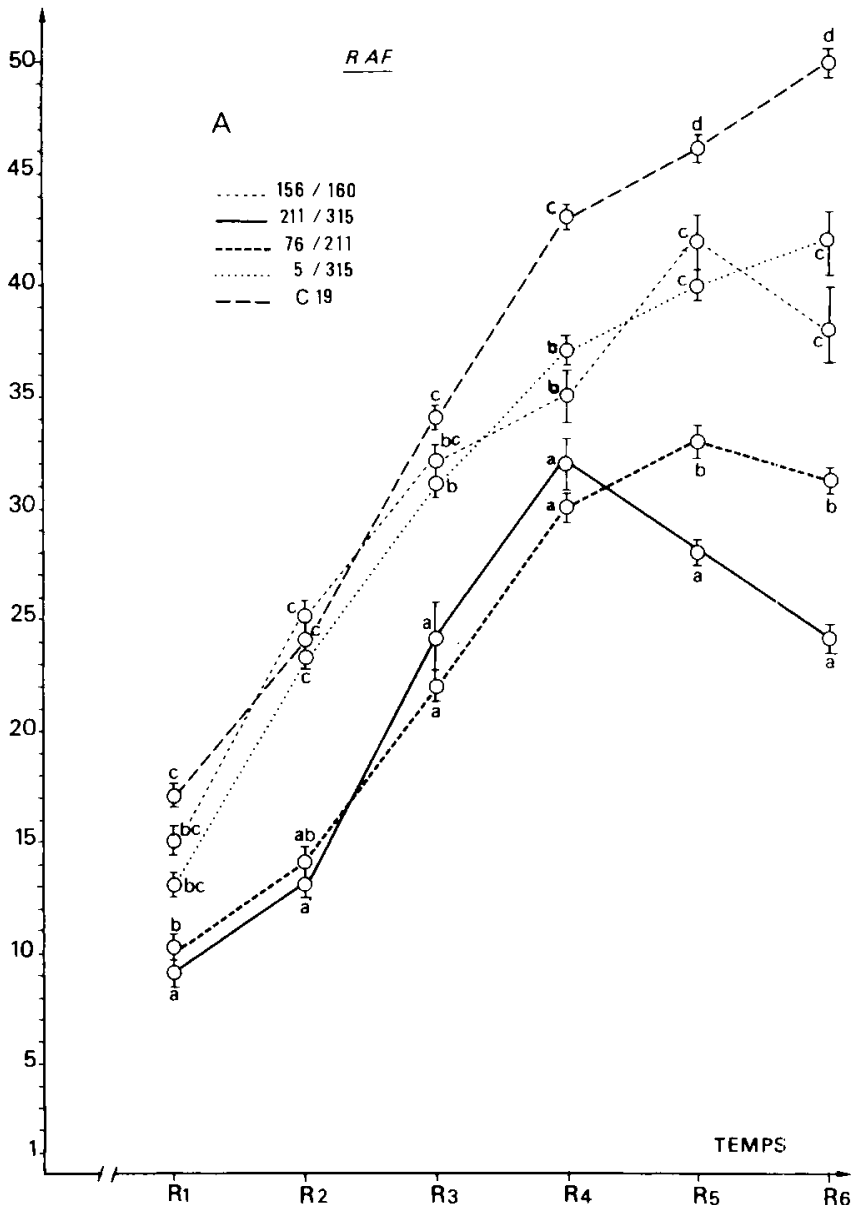

EXTENSION DES SYMPTOMES SUR LA TIGE

EN \% DE LA TAILLE

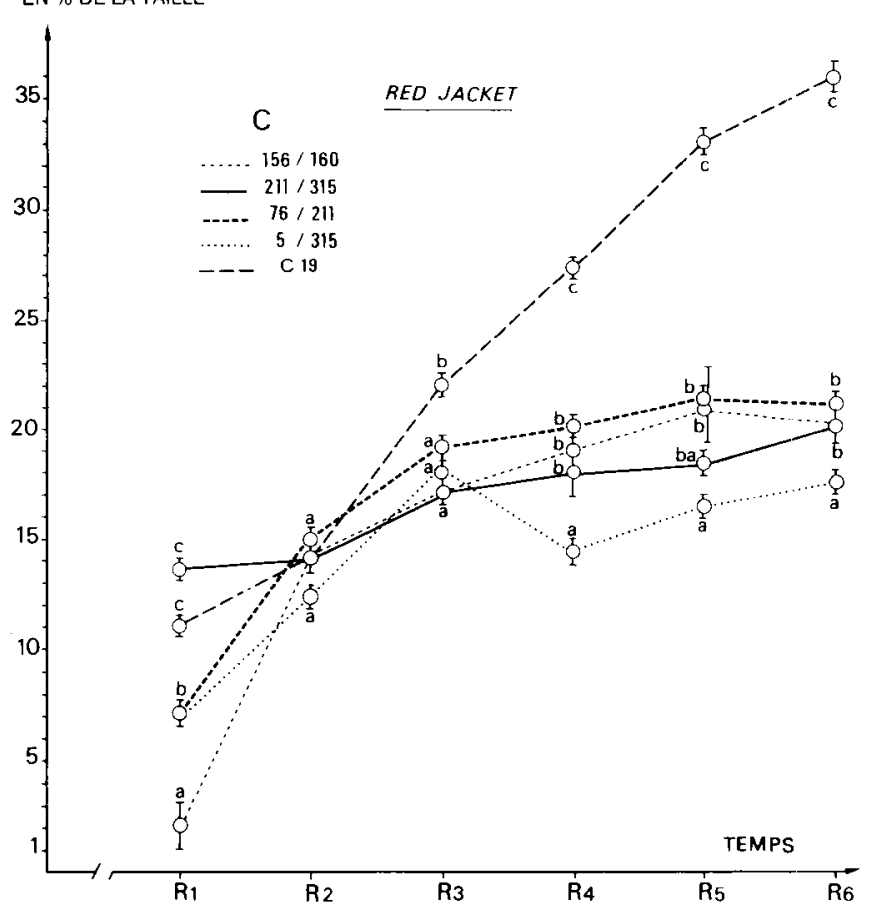

Figures 1A, 1B, 1C, 1D

Indice d'extension des symptômes foliaires : pourcentage du maximum d'extension possible.

Intervalles de confiance au seuil de $95 \mathrm{p} .100$.

$\mathrm{a}, \mathrm{b}, \mathrm{c}, \mathrm{d}$ : groupes de valeurs déterminés par le test de Duncan pour chaque relevé.

$R I$ à $R 6$ : relevés hebdomadaires.
EXTENSION DES SYMPTOMES SUR LA TIGE

EN \% DE LA TAILLE

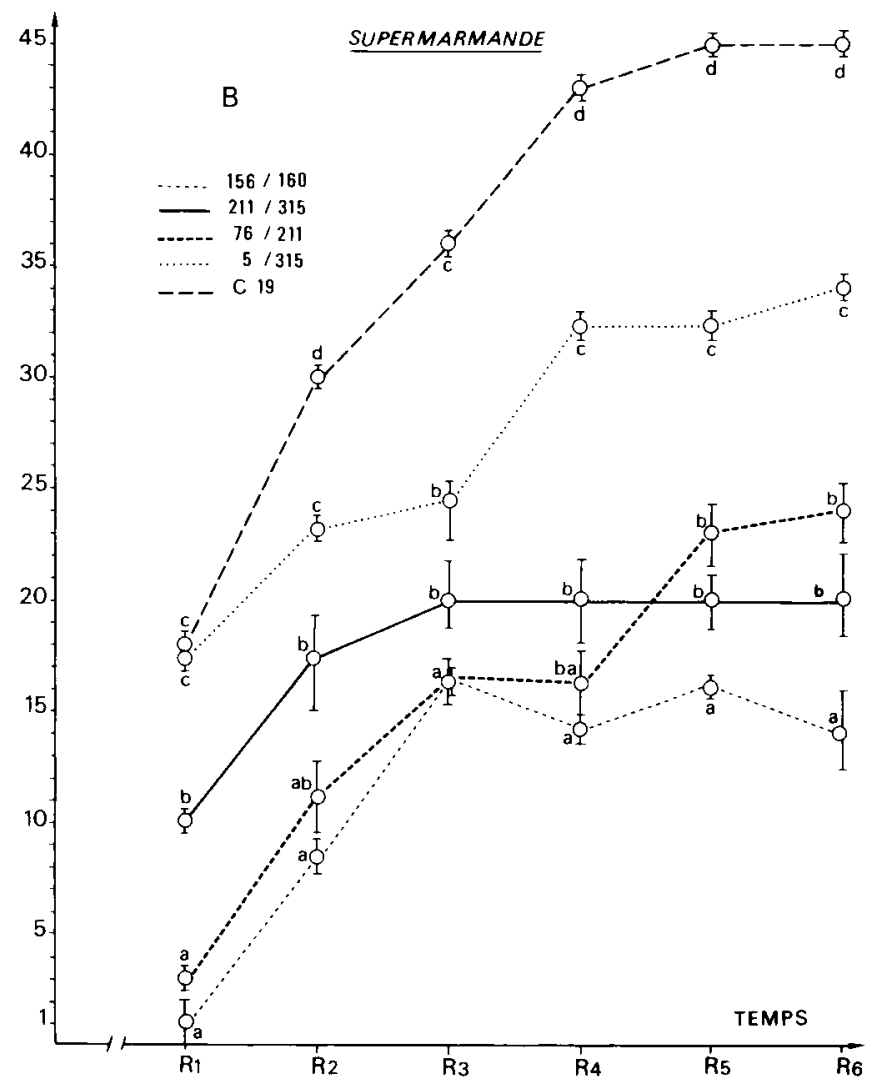

EXTENSION DES SYMPTOMES SUR LA TIGE

EN \% DE LA TAILLE

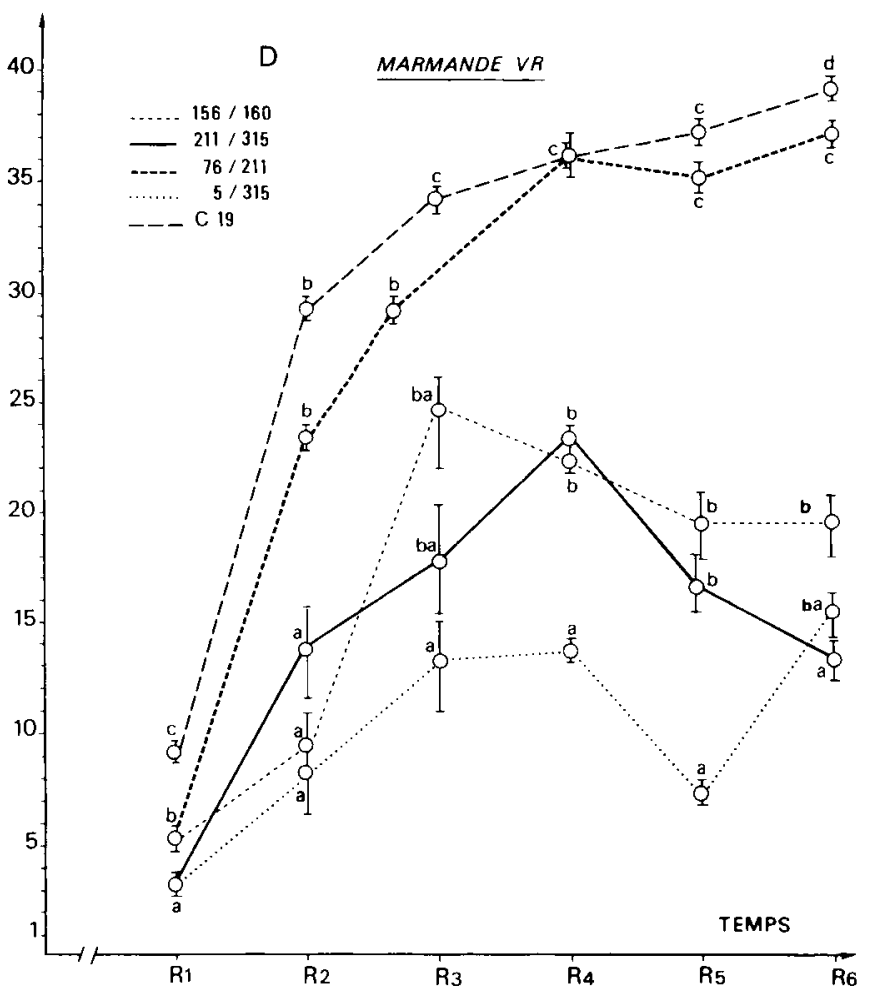

Extent of leaf symptoms : percent of maximum possible extent.

$95 \%$ confidence intervals.

$\mathrm{a}, \mathrm{b}, \mathrm{c}, \mathrm{d}$ : value groups as determined by the Duncan's multiple range test for each reading.

$R I$ to $R 6$ : weekly readings. 
sive à l'encontre d'une autre. Les classements des lignées en fonction des variétés apparaissent en lettres minuscules au tableau la. C'est ainsi que la lignée «76/211» fait apparaître les premiers signes de la maladie bien avant toutes les autres chez la variété "Raf "; elle est en revanche une des moins efficaces chez les variétés «Supermarmande » et «Marmande VR ». A l'inverse, la lignée « 211/315» provoque les dommages les plus tardifs chez la variété « Raf » et les plus précoces chez la variété « Red Jacket ».

Si réciproquement, la durée de la période d'incubation est considérée comme l'une des expressions de la résistance générale de la tomate à la verticilliose, une même variété peut se classer comme la moins sensible lorsqu'elle est infectée par une lignée de $V$. dahliae et comme la plus sensible lorsqu'elle héberge une autre lignée (classements en lettres majuscules au tableau la). La variété " Marmande VR » est dans les dernières à manifester la maladie en présence des lignées « C19 » ou « 211/315», mais dans les premières lorsqu'elle est confrontée à la lignée « 5/315».

De même, la variété « Raf » est une des plus résistantes aux attaques de la lignée « 211/315» et la plus sensible à celles des lignées «76/211》 et «156/160》.

Ces changements de classement statistiquement significatifs résultent d'interactions spécifiques entre des variétés et des lignées déterminées, comme le montre l'analyse de variance (tabl. 1b).

\section{B. Extension des symptômes foliaires (fig. 1A à 1D)}

La progression des symptômes de la verticilliose en direction du sommet de la tige est indiquée en valeur relative. C'est la portion relative de tige visiblement malade par rapport à la hauteur totale de la plante. L'indice ainsi obtenu intègre, d'une part, le fait que la croissance en hauteur de la plante peut être altérée par l'agent pathogène, et, d'autre part, le fait que cette croissance ne se poursuit pas nécessairement à la même vitesse tout au long des observations. Sous ces réserves, les réponses des 4 variétés à l'inoculation des 5 lignées présentent des différences marquées.

Au cours des 2 semaines qui suivent la fin de la période d'incubation, la variété "Raf " (fig. 1A) permet de distinguer 2 groupes de lignées, l'un moins agressif («211/315» et «76/211»), l'autre plus agressif («156/160», «5/315» et «C19»). Par la suite 3 groupes peuvent être reconnus. L'un, constitué des lignées « 211/315 » et « 76/211 », se caractérise par l'arrêt de l'extension des dégâts vers le sommet. Pour le second groupe des symptômes progressent au moins à la vitesse de croissance de l'axe aérien. Le troisième ne comprend que la lignée « C19»: les dommages s'étendent nettement plus vite que la tige ne croît. De même, sur la variété "Supermarmande " (fig. 1B), aucune lignée recombinée n'approche de la lignée sauvage « C19 ». La lignée « 156/160» est constamment la moins performante pendant toute la durée des observations tandis que la lignée « $5 / 315$ » est régulièrement la plus performante des lignées recombinées.

Chez la variété « Red Jacket » (fig. 1C), l'extension des symptômes de la verticilliose en direction du sommet de la plante est également et simultanément freinée quand ils sont provoqués par l'inoculation de l'une des 4 lignées recombinées. En revanche cette extension se poursuit pendant toute la durée des observations sur les plantes de cette même variété inoculées avec la lignée «C19».

Quant à la variété «Marmande VR » (fig. 1D) elle classe les lignées en 2 groupes très distincts : le premier, plus agressif, comprend la lignée « C19 » et la lignée recombinée « $76 / 211$ » tandis que les 3 autres lignées forment le second groupe moins agressif.

Enfin, des interactions spécifiques variétés $\times$ lignées se traduisent par des différences de classement. Ainsi, la lignée «76/211» dont l'agressivité est forte et proche de celle de la lignée "C19 » chez la «Marmande VR », se range parmi les moins agressives à l'égard des variétés «Raf » et « Supermarmande ». De même, les lignées « 5/315 » et « 156/160», qui sont les plus dangereuses - après la lignée « C19 » - pour la variété "Raf», sont les moins nocives pour les variétés « Marmande VR » et «Supermarmande » respectivement.

\section{Intensité des altérations foliaires (fig. 2A à 2D)}

Chez la variété « Raf » (fig. 2A), la lignée sauvage «C19» ne se montre la plus agressive qu'en fin d'expérience. A l'opposé, la lignée «211/315» est constamment la moins agressive.

Inoculée à la variété "Supermarmande » (fig. 2B), la lignée sauvage se montre la plus agressive tout au long de la période d'observation : la gravité des dommages foliaires s'accentue jusqu'à la fin de l'expérience. Les 4 lignées recombinées en revanche ne se séparent en 2 groupes que lors de la dernière notation, les lignées « $5 / 315$ » et « $76 / 211$ » provoquant finalement plus d'altérations que les lignées « $156 / 160$ » et «211/315».

La variété « Red Jacket » (fig. 2C) réagit à l'inoculation de la lignée «C19 » d'une manière analogue à celle de la variété " Raf ». Cette lignée "C19» ne se distingue de toutes les autres par sa plus forte agressivité qu'après plusieurs semaines d'observation. Sur la variété « Red Jacket », les 4 lignées recombinées ont un comportement très voisin ; au contraire de leur attitude sur la variété " Raf » où les indices d'altérations foliaires en fin d'observation diffèrent davantage d'une lignée à l'autre.

La variété «Marmande VR » (fig. 2D) est, comme la variété "Supermarmande ", très tôt plus gravement endommagée par la lignée «C19» que par les 4 autres. Mais, ces autres lignées se comportent différemment. La lignée « 211/315 » est plus agressive que la lignée «5/315» vis-à-vis de la «Marmande VR»; c'est l'inverse sur la «Supermarmande ». Il apparaît ainsi que l'évolution des altérations foliaires chez une variété présente des modalités différentes selon les lignées de $\boldsymbol{V}$. dahliae et que, réciproquement, le pouvoir pathogène d'une même lignée s'exprime différemment d'une variété de tomate à l'autre.

\section{La croissance de l'axe aérien (fig. 3A à 3D)}

Les variétés « Raf » (fig. 3A) et « Marmande VR » (fig. 3D) répondent soit par une augmentation de leur croissance soit par un rabougrissement selon la lignée 

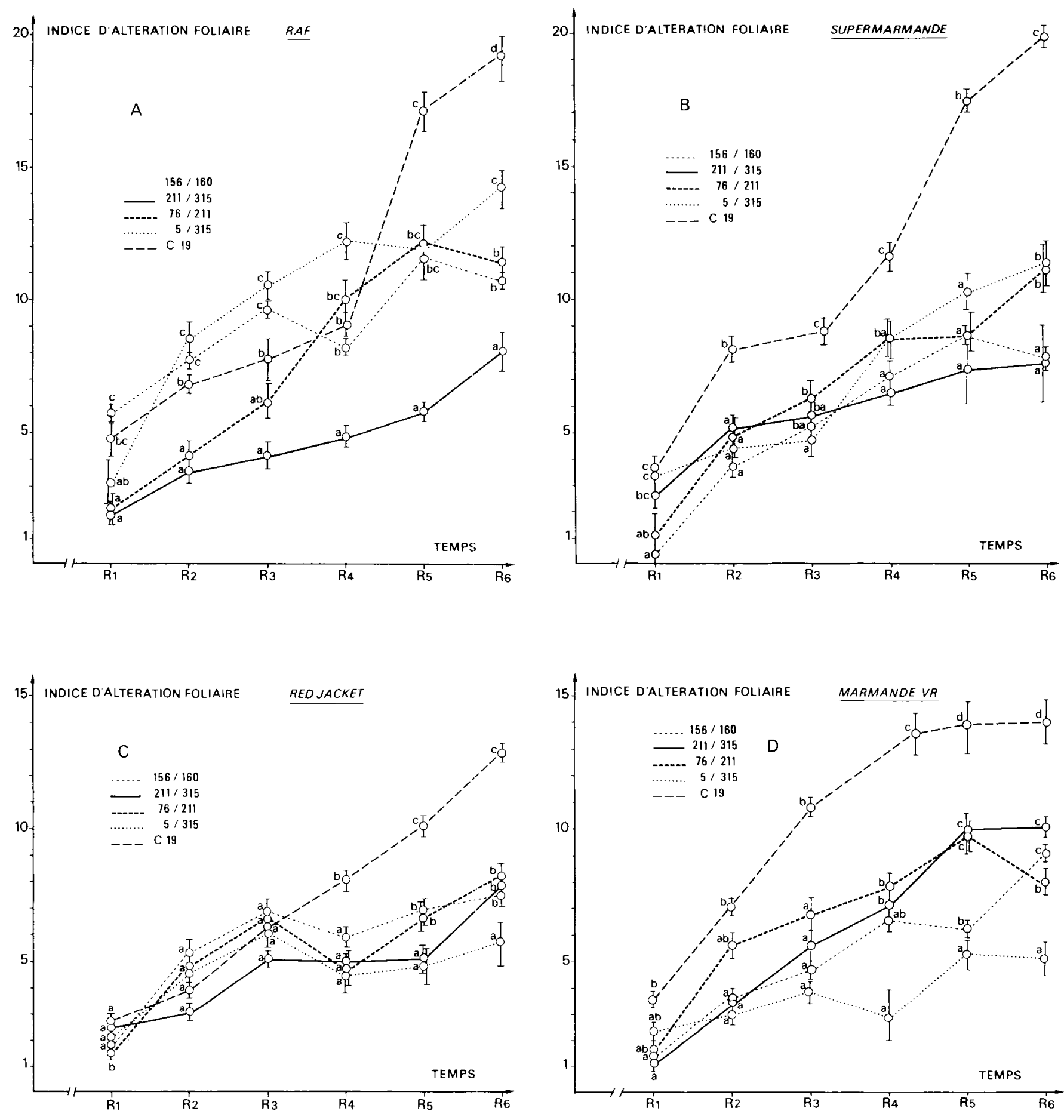

Figures 2A, 2B, 2C, 2D

Evolution des indices d'altération foliaire.

Intervalles de confiance au seuil de $95 \mathrm{p} .100$.

$\mathrm{a}, \mathrm{b}, \mathrm{c}, \mathrm{d}$ : groupes de valeurs déterminés par le test de Duncan pour chaque relevé.

RI à R6: relevés hebdomadaires.

inoculée. Ce n'est pas la même lignée qui entrâne un surcroît d'élongation chez les 2 variétés (" 156/160" chez la variété " Raf »; «76/211» pour la variété «Marmande VR »).

La variété «Supermarmande» (fig. 3B), contaminée par la lignée « $5 / 315$ », présente un fort accroissement de la longueur de son axe aérien au début des observations, mais elle retrouve bientôt une hauteur comparable à celle des témoins non inoculés. Cette situation ne s'observe qu'au niveau de ce couple. Pour ce qui a trait à l'effet dépresseur sur la croissance, cette dernière variété se classe comme la plus sensible ; 3 lignées recombinées (« 211/315», «76/211», ( $156 / 160 »)$ sont plus agressives que la sauvage "C19 ». A l'inverse, chez les 3 autres variétés, cette lignée sauvage « $\mathrm{C} 19$ » est des plus inhibitrices. 

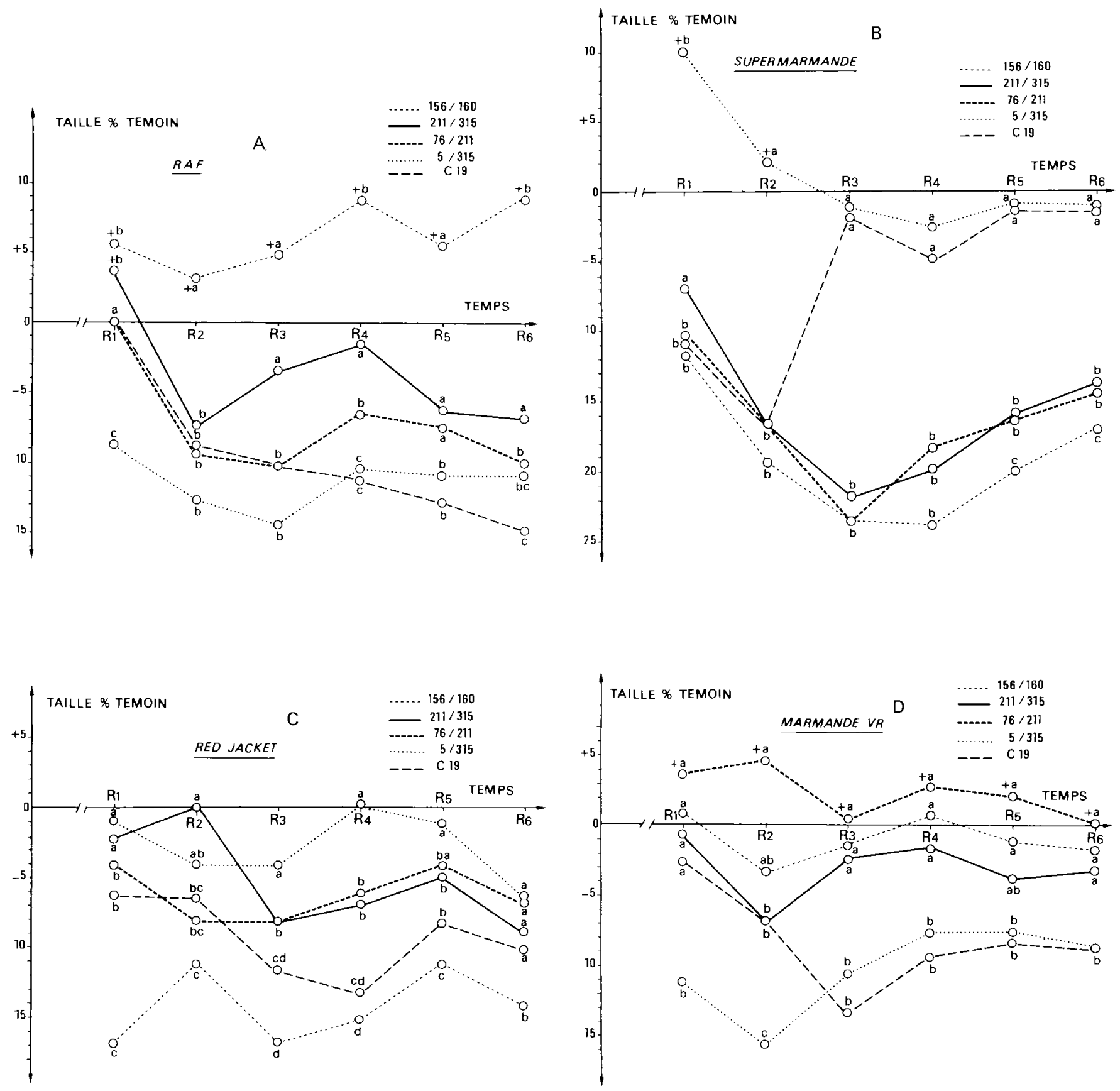

Figures 3A, 3B, 3C, 3D

Variations de la hauteur de l'axe aérien des plantes contaminées, par rapport au témoin de la même variété.

Le rapport (taille des traités - taille des témoins)/(taille des témoins) $\times 100$ est en ordonnée.

$\mathrm{a}, \mathrm{b}, \mathrm{c}, \mathrm{d}:$ groupes de valeurs déterminés par le test de Duncan pour chaque relevé.

$R I$ à $R 6$ : relevés hebdomadaires.

La variété « Red Jacket» (fig. 3C) manifeste toujours la maladie par une diminution de la hauteur de l'axe aérien quelle que soit la lignée inoculée.

Les interactions spécifiques variété $\times$ lignée s'illustrent par des exemples tels que les effets de la lignée "C19» sur la variété "Supermarmande " (où «C19» est moins efficace que les lignées « 211/315», «76/211 », «156/160 ») et sur la variété « Raf » (où
Height of the inoculated plants as a percentage of the control. The ratio (inoculated plant height - control height)/(control height) $\times 100$ is the ordinate.

$\mathrm{a}, \mathrm{b}, \mathrm{c}, \mathrm{d}$ : value groups as determined by the Duncan's multiple range test for each reading.

$R 1$ to $R 6$ : weekly readings.

« C19 » est plus efficace que ces mêmes lignées). La lignée «156/160 » illustre aussi ces interactions spécifiques. Cette lignée présente le plus fort effet dépresseur sur l'accroissement de la tige des variétés " Red Jacket » et " Supermarmande ». Par contre, elle est de celles qui affectent le moins la taille de la variété « Marmande VR » et elle accroît même la hauteur des plantes de la variété « Raf ». 


\section{E. Le rythme d'émission foliaire (fig. 4A à 4D)}

Chez la variété «Raf» (fig. 4A), l'inoculation de $V$. dahliae se traduit par une stimulation de l'émission des feuilles pendant toute la durée de l'expérience (lignée « 156/160») ou seulement à son début (lignée « 211/315»). Quant aux lignées «76/211 » et « $5 / 315$ », elles entraînent très tôt une nette dépression du développement foliaire. La lignée « C19 » présente un comportement intermédiaire : légèrement stimulante lors de la fin de la période d'incubation, elle est ensuite fortement inhibitrice.

Chez la variété "Supermarmande » (fig. 4B), toutes les lignées de $V$. dahliae entraînent très tôt une rette dépression du développement foliaire. Cette réduction est profonde et durable lorsqu'elle est provoquée par les lignées « 156/160», «76/211», «211/315», plus
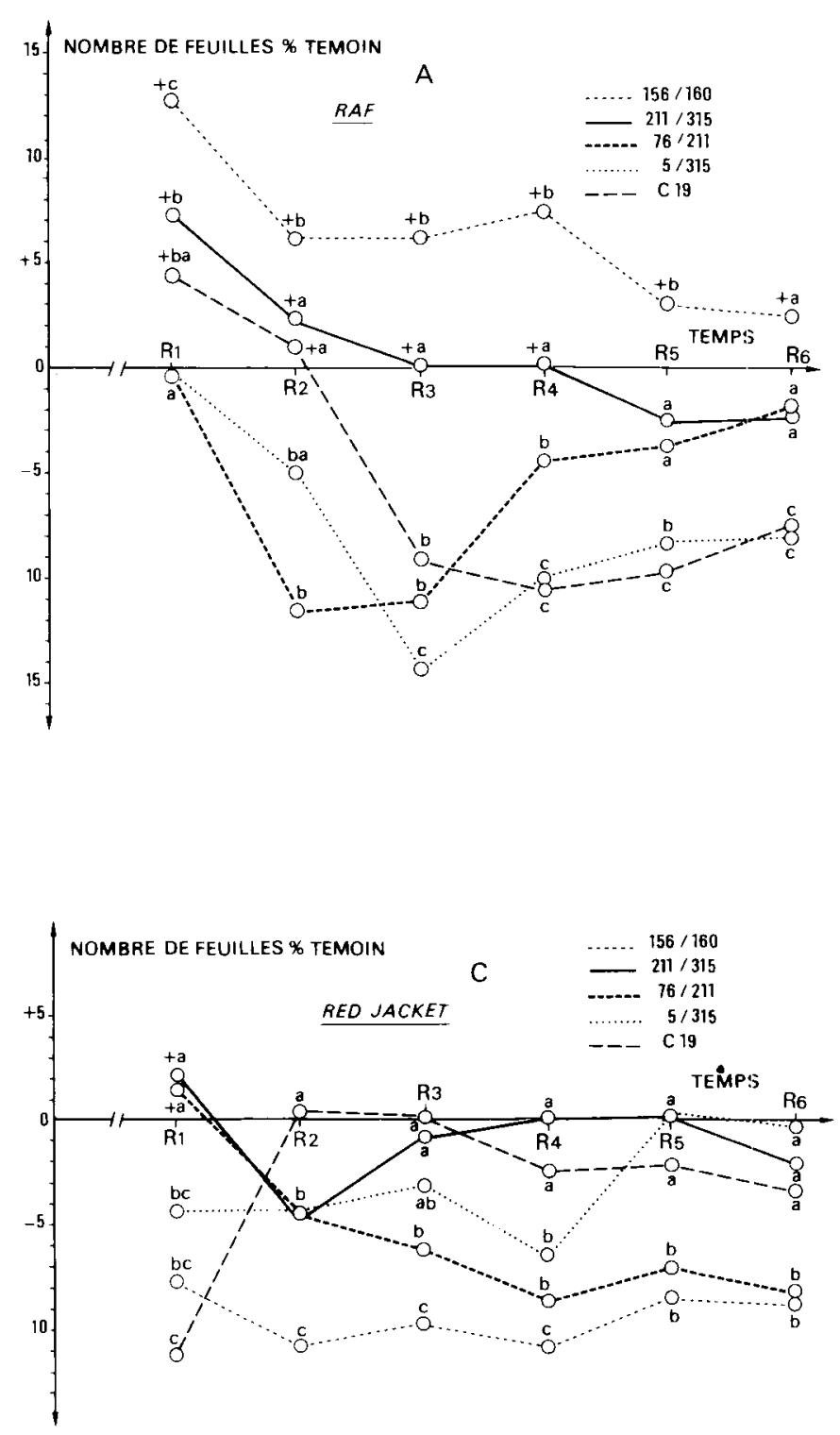

Figures 4A, 4B, 4C, 4D

Variation du nombre de feuilles émises par les plantes contaminées, en p. 100 du témoin.

Le rapport (nbre feuilles du traité - nbre feuilles du témoin)/(nbre feuilles du témoin) $\times 100$ est en ordonnée.

$\mathrm{a}, \mathrm{b}, \mathrm{c}, \mathrm{d}$ : groupes de valeurs déterminés par le test de Duncan pour chaque relevé.

$R I$ à $R 6$ : relevés hebdomadaires. légère mais permanente dans le cas de la lignée " C19 », plus faible encore et temporaire dans le cas de la lignée « $5 / 315 »$.

La diminution de l'émission des feuilles est précoce chez la variété « Red Jacket » (fig. 4C). Elle persiste et est notable lorsque les inoculations sont effectuées avec les lignées «156/160 » et «76/211》. Elle est moins marquée et s'atténue avec le temps après inoculation des lignées « C19 » et « 211/315 » ou même disparaît (lignée « $5 / 315$ »). La variété «Marmande VR » (fig. 4D) réagit tantôt par une accélération de l'émission des feuilles (lignées «76/211 》 et «156/160») suivie d'un ralentissement, tantôt par une diminution profonde mais temporaire (lignée «211/315»), durable et importante (lignée «C19») ou peu marquée (lignée « $5 / 315 »)$.
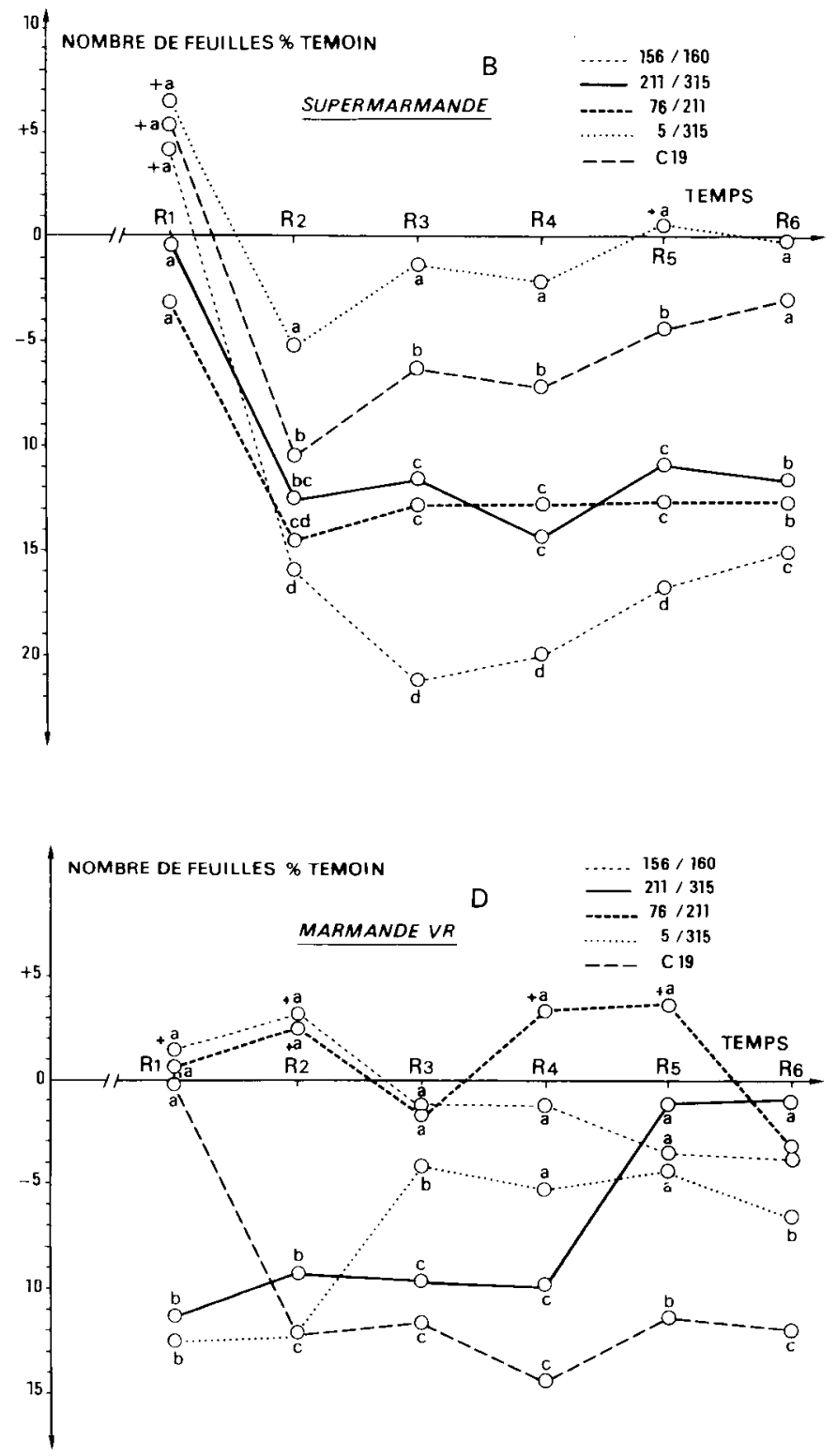

Variation in the number of leaves on inoculated plants as a percentage of the number in the control.

The ratio (number of leaves on inoculated plants - number of leaves on control) $/($ number of leaves on control) $\times 100$ is the ordinate. $\mathrm{a}, \mathrm{b}, \mathrm{c}, \mathrm{d}$ : value groups as determined by the Duncan's multiple range test for each reading.

$R I$ to $R 6$ : weekly readings. 
Pour cette caractéristique de la verticilliose comme pour les précédentes, l'existence d'interactions spécifiques entre les variétés et les lignées se manifeste par des changements de classement ou même par l'inversion des effets de la maladie. Ainsi la lignée " 156/160" entraîne une très nette diminution du nombre de feuilles chez la variété "Supermarmande » et une légère augmentation chez la variété " Raf ». Ici encore, la lignée « C19 » est la plus agressive à l'égard des variétés "Raf » et " Marmande VR » mais l'est beaucoup plus modérément à l'égard des variétés « Red Jacket 》 et « Supermarmande».

\section{F. Floraison (tabl. 2)}

Pour toutes les variétés, la floraison est réduite chez les plantes contaminées. La variété " Raf » ne distin- gue pas les 5 lignées par leur effet sur sa floraison : le nombre de fleurs est sensiblement le même avec toutes les lignées.

Les 3 autres variétés de tomate ne classent pas nécessairement dans le même ordre les différentes lignées du parasite (lettres minuscules du tableau 2a). Les lignées «76/211 » (classée $b$ ou $c$ ) et " 5/315» (classée $a$ ou $a b$ ) sont aux 2 extrémités de l'échelle d'agressivité chez les 3 variétés. Mais chez «Supermarmande » et « Red Jacket ", la lignée «156/160" (classée $a b$ et $b c$ ) est moins agressive que chez "Marmande VR " (où elle est classée $c$ ). Chez «Red Jacket", la lignée «211/315» est peu agressive; elle l'est davantage chez « Marmande VR » et encore plus chez "Supermarmande ».

Réciproquement, les différentes lignées de $V$. dahliae ne classent pas les différentes variétés de tomate dans un ordre immuable (lettres majuscules du

TABLEAU 2

Nombre de fleurs portées par les 3 premières inflorescences, pour les 864 plantes.

Flower count on the 3 first inflorescences, for the 864 plants.

TABLEAU $2 \mathrm{a}$

Test de rang de DUNCAN (niveau 5 p. 100) du nombre moyen de fleurs, en fonction des variétés et des lignées. L'effectif de chaque classe est de 36.

$\mathrm{a}, \mathrm{b}, \mathrm{c}, \mathrm{d}$ : classement des lignées par variété : à lire verticalement.

$\mathrm{A}, \mathrm{B}, \mathrm{C}$ : classement des variétés par lignée : à lire horizontalement.

DUNCAN multiple range test (5\% level) of average flower number for different varieties and strains. Each class size is 36.

$\mathrm{a}, \mathrm{b}, \mathrm{c}, \mathrm{d}$ : classification of strains by variety : read vertically.

A, B, C : classification of varieties by strain : read horizontally.

\begin{tabular}{|c|c|c|c|c|c|c|c|c|}
\hline \multirow[t]{2}{*}{ Lignées } & \multicolumn{8}{|c|}{ Variétés } \\
\hline & & Raf & & marmande & & ed Jacket & & mande VR \\
\hline \multirow{2}{*}{$\mathrm{C} 19$} & & A & & $\mathrm{A}$ & & A & & A \\
\hline & a & $7,0 \pm 1,1$ & b & $7,2, \pm 1,0$ & $b c$ & $7,8 \pm 1,4$ & $c$ & $6,9 \pm 0,8$ \\
\hline \multirow{2}{*}{$76 / 211$} & & A & & A & & A & & A \\
\hline & $\mathrm{a}$ & $6,9 \pm 0,8$ & $\mathrm{~b}$ & $7,3 \pm 1,0$ & $\mathrm{c}$ & $7,3 \pm 1,3$ & $\mathrm{c}$ & $6,3 \pm 1,2$ \\
\hline \multirow{2}{*}{$5 / 315$} & & B & & $\mathrm{AB}$ & & $\mathrm{A}$ & & A \\
\hline & a & $7,1 \pm 1,0$ & $\mathrm{a}$ & $9,1 \pm 1,3$ & $a b$ & $9,3 \pm 1,2$ & a & $10,6 \pm 1,3$ \\
\hline \multirow{2}{*}{$211 / 315$} & & $\mathrm{C}$ & & $\mathrm{BC}$ & & A & & $\mathrm{AB}$ \\
\hline & a & $7,0 \pm 1,2$ & $b$ & $7,4 \pm 1,3$ & $a b$ & $9,3 \pm 1,1$ & $b$ & $8,7 \pm 1,1$ \\
\hline \multirow{2}{*}{$156 / 160$} & & $\mathrm{AB}$ & & $\mathrm{A}^{\bullet}$ & & B & & B \\
\hline & a & $7,1 \pm 1,2$ & $a b$ & $8,4 \pm 1,1$ & $\mathrm{bc}$ & $8,1 \pm 1,3$ & $\mathrm{c}$ & $5,4 \pm 1,6$ \\
\hline Témoins & & $11,2 \pm 1,3$ & & $11,5 \pm 1,1$ & & $10,2 \pm 0,7$ & & $12,8 \pm 1,3$ \\
\hline
\end{tabular}

TABLEAU $2 \mathrm{~b}$

Analyse de variance à 2 critères de classification (variété $\times$ lignée).

$n$ : nombre de variétés de tomate ; $p$ : nombre de lignées de Verticillium.

Two way analysis of variance ( $n$ varieties $\times p$ strains).

\begin{tabular}{ccccrc}
\hline \hline Source de variation & \multicolumn{2}{c}{ Degrés de liberté } & Carré moyen & F & Signification de F \\
\hline Variété & 3 & $(\mathrm{n}-1)$ & 38,152 & 3,211 & 0,022 \\
Lignée + témoin & 5 & $(\mathrm{p}-1)$ & 418,713 & 35,243 & $<0,001$ \\
Interac. Var. $\times$ lign. & 15 & $(\mathrm{n}-1)(\mathrm{p}-1)$ & 33,939 & 3,362 & $<0,001$ \\
Total expliqué & 23 & $(\mathrm{np}-1)$ & 122,048 & 10,273 & $<0,001$ \\
Résiduelle & & 840 & 11,881 & & \\
\hline \hline
\end{tabular}


tableau 2a). Pour les lignées « C19 » et « 76/211 », les 4 variétés de tomate sont toutes également sensibles. En outre, «Red Jacket » se classe toujours dans les plus sensibles. Mais "Supermarmande " et "Raf " sont plus sensibles à la lignée «211/315» qu'à « 5/315» ou « 156/160 ». Enfin, " Marmande VR » qui résiste bien à « $5 / 315$ » se retrouve la plus sensible à $《 156 / 160$ ».

L'analyse de variance montre que l'interaction souche $\times$ variété est très significative (tabl. $2 b$ ).

\section{DISCUSSION ET CONCLUSION}

Cette étude montre que les 4 variétés de tomate utilisées sont sensibles à toutes les lignées de Verticillium utilisées. Les lignées « C19 » et ses dérivées apparaissent donc capables de contourner la résistance conférée par le gène Ve présent chez « Marmande VR ». Il n'y a pas d'interactions qualitatives différentielles (maladie/ non-maladie) entre l'ensemble des lignées du parasite et l'ensemble des variétés de l'hôte.

Par contre, on constate qu'une lignée de Verticillium dahliae peut se montrer très efficace pour induire une des manifestations de la verticilliose et très peu apte à provoquer d'autres manifestations. C'est ainsi que la lignée « $5 / 315$ » qui perturbe très peu l'élongation de l'axe aérien et l'émission foliaire de la variété « Supermarmande " est très agressive si l'on considère le degré d'altération des feuilles, et donc de la fonction chlorophyllienne, et l'extension rapide des symptômes sur les parties visibles de la plante. Inversement une même variété de tomate peut exprimer intensément certains signes de la maladie, et d'autres plus faiblement. Un exemple est celui des variétés " Marmande VR » ou « Red Jacket » qui manifestent et laissent progresser le moins les altérations foliaires tandis qu'elles réagissent autant que les autres variétés sur les autres manifestations de la verticilliose. Si l'on prend en compte comparativement plusieurs manifestations de la verticilliose chez la tomate on peut faire les constatations qui suivent :

- aucune variété ne répond exactement de la mème façon aux attaques des différentes lignées de Verticillium dahliae ;

- aucune lignée ne provoque des dommages strictement identiques chez toutes les variétés.

Certes, les différences peuvent porter sur la nature de certains symptômes : la lignée «156/160》 provoque un rabougrissement sur la variété "Supermarmande " et un relatif gigantisme sur la variété "Raf »; " 76/211" accroît un peu le nombre de feuilles sur la variété « Marmande VR » et le diminue sur les 3 autres variétés. Mais ici, comme pour chacune des autres manifestations de la maladie prise individuellement, les différences entre souches comme entre variétés sont finalement mesurables en termes quantitatifs. Ainsi, dans le cas de la verticilliose de la tomate, chez les variétés étudiées vis-à-vis d'un groupe déterminé de lignées de parasite, on observe des interactions spécifiques entre hôte et agent pathogène, au moins pour une part des éléments contribuant à l'agressivité et à la résistance générale. Ceci nous amène à considérer ces éléments comme des composantes de résistance chez l'hôte auxquelles correspondent des composantes d'agressivité chez le parasite. Ces composantes sont bien distinctes puisque les effets varient d'un hôte à l'autre et aussi d'une lignée parasite à l'autre, ce qui se traduit précisément par les interactions observées.

Une explication possible est de considérer la résistance générale comme la manifestation d'une somme de gènes mineurs contrôlant ensemble une résistance partielle, mais qui interagissent spécifiquement avec des gènes complémentaires gouvernant l'agressivité chez le parasite. D'ailleurs, des travaux ont montré des possibilités d'expression quantitative dans les relations verticales (CLIFFORD \& CLOTHIER, 1974 ; CROSSE, 1975 ) et NELSON (1978) a décrit ce qu'il appelle des «situations intermédiaires" dans le domaine de la résistance horizontale. Finalement, on peut imaginer la résistance générale comme constituée d'une somme de résistances spécifiques (sensu VAN DER PLANK) contrôlées par des gènes mineurs. La faiblesse des effets individuels des gènes impliqués et l'importance de l'additivité et des effets épistasiques qui existent entre eux permettraient éventuellement d'expliquer pourquoi ce type de résistance a été perçu à l'origine (VAN DER PLANK, 1968) comme non spécifique et non variable.

Dans ces conditions de telles interactions spécifiques pourraient, comme dans le cas de la résistance verticale, être à l'origine d'une pression sélective sur les populations du pathogène. Cette pression, si elle est constamment orientée dans le même sens par l'emploi continu de la même variété de l'hôte, pourrait conduire à des changements dans la structure des populations pathogènes : les génotypes favorisés finiraient par prédominer. Ainsi, le fait que toutes les lignées soient capables d'induire la maladie n'empêche pas que la structure de la population pathogène puisse évoluer en fonction de la structure de la population hôte. Les changements qui interviennent au niveau des populations pathogènes pourraient bien alors se traduire par une érosion, une chute du niveau originel de résistance générale. On ne peut pour autant, imaginer que la résistance générale s'effondre totalement, en raison de l'homéostase génétique, des avantages attachés aux états moyens aux dépens des extrêmes. Cependant, signalons aussi qu'une souche de parasite n'a pas besoin de l'agressivité optimale sur toutes les manifestations de la maladie pour que la déficience de la résistance horizontale ait une incidence remarquable sur la production.

Dans cette perspective, la mise en compétition de 2 lignées pathogènes qui induisent des manifestations différentes de la verticilliose chez 2 variétés hôte et la mesure de l'inoculum secondaire, produit dans des conditions standard, pourraient donner des indications sur l'existence et les effets d'une pression de sélection de la résistance horizontale de l'hôte sur l'agressivité du pathogène. Cet aspect est étudié dans un prochain article.

Reçu le 5 mars 1987. Accepté le 21 février 1988.

\section{REMERCIEMENTS}

Les auteurs remercient Monsieur le Professeur. Chevaugeon pour ses nombreux avis et conseils dans la préparation et la réalisation du texte. Ils remercient également Monsieur TAllLER pour son aide technique ainsi que Monsieur Guénard des Ets Clause pour la fourniture des semences. 


\section{RÉFÉRENCES BIBLIOGRAPHIQUES}

Beye I., Lafay J.-F., 1985. Etude de critères de sélection pour une résistance générale à la verticilliose chez la tomate. Agronomie, $\mathbf{5}$, 305-311.

Chevaugeon J., 1979. Phytophthora infestans : un exemple d'intervention de l'homme sur la structure d'une espèce parasite. Bull. Soc. bot. Fr., Actual. bot., 126, 21-44.

Clifford B. C., Clothier R. B., 1974. Physiological specialization of Puccinia hordei on barley hosts with non-hypersensitive resistance. Trans. Br. mycol. Soc., 63, 421-430.

Crosse J. E., 1975. Variation amongst plant pathogenic bacteria. Ann. Appl. Biol., 81, 438.

Duncan D. B., 1955. Multiple range and multiple F-test. Biometrics, 11, 1-42.

Flor H. H., 1956. The complementary genic systems in flax and flax rust. $A d v$. Genet., 8, 29-54.

Martin T. J., Elingboe A. H., 1976. Differences between compatible parasite/host genotypes involving the Pm4 locus of wheat and the corresponding genes in Erysiphe graminis f. sp. tritici. Phytopatho$\log y$, 66, 1435-1438.
Nelson R. R., 1978. Genetics of horizontal resistance to plant diseases. Ann. Rev. Phytopathol., 16, 359-378.

Parlevliet J. E., 1976. Evaluation of the concept of horizontal resistance in the barley/Puccinia hordei host-pathogen relationship. Phytopathology, 66, 494-497.

Parlevliet J. E., Zadoks J. C., 1977. The integrated concept of disease resistance : a new view including horizontal and vertical resistance in plants. Euphytica, 26, 5-21.

Sebti S., 1982. Essai d'analyse des composantes du pouvoir pathogène de Verticillium albo-atrum Reinke et Berthold. Thèse Doct. $3^{\text {e }}$ Cycle, Univ. Paris-Sud, Orsay.

Skajennikoff M., Rapilly F., 1983. Etude sur l'agressivité de Septoria nodorum Berk. : Effet des hôtes (triticale et blé) et des organes attaqués. Agronomie, 3, 131-140.

Van der Plank J. E., 1968. Disease resistance in plants. Acad. Press Inc. New York, London, $204 \mathrm{p}$.

Van der Plank J. E., 1978. Genetic and molecular basis of plant pathogenesis. Springer-Verlag. Berlin, Heidelberg, New York, $167 \mathrm{p}$. 